\title{
On Products of Distributions in Colombeau Algebra
}

\author{
Marija Miteva, ${ }^{1}$ Biljana Jolevska-Tuneska, ${ }^{2}$ and Tatjana Atanasova-Pacemska ${ }^{1}$ \\ ${ }^{1}$ Faculty of Computer Sciences, University of Goce Delcev, Krste Misirkov Street br 10A, 2000 Stip, Macedonia \\ ${ }^{2}$ Faculty of Electrical Engineering and Informational Technologies, University Ss. Cyril and Methodius in Skopje, \\ Karpos II bb, 1000 Skopje, Macedonia
}

Correspondence should be addressed to Biljana Jolevska-Tuneska; biljanaj@feit.ukim.edu.mk

Received 2 December 2013; Accepted 12 January 2014; Published 23 February 2014

Academic Editor: Sotiris Ntouyas

Copyright (c) 2014 Marija Miteva et al. This is an open access article distributed under the Creative Commons Attribution License, which permits unrestricted use, distribution, and reproduction in any medium, provided the original work is properly cited.

Results on products of distributions $x_{+}^{-k}$ and $\delta^{(p)}(x)$ are derived. They are obtained in Colombeau differential algebra $\mathscr{G}(\mathbf{R})$ of generalized functions that contains the space $\mathscr{D}^{\prime}(\mathbf{R})$ of Schwartz distributions as a subspace. Products of this form are useful in quantum renormalization theory in Physics.

\section{Introduction}

In quantum physics one find the need to evaluate $\delta^{2}$, when calculating the transition rates of certain particle interactions see [1]. The problem of defining products of distributions is also closely connected with the problem of renormalization in quantum field theory. Due to the large use of distributions in the natural sciences and other mathematical fields, the problem of the product of distributions [2] is an objective of many research studies. Starting with the historically first construction of distributional multiplication by König [3] and the sequential approach developed in [4] by Antosik et al., there have been numerous attempts to define products of distributions, see [5-7], or rather to enlarge the number of existing products.

In order to define a general product of distributions the mathematicians were seeking an algebra $A$ containing $\mathscr{D}^{\prime}(\mathbf{R})$, having the constant function 1 as a unit element and such that there is a derivation $D: A \rightarrow A$ which is linear, which generalizes the derivation in the sense of distributions and such that Leibnitz's rule for derivation of a product holds. With his famous impossibility result Schwartz [8] showed that the new product in $A$ cannot coincide with the usual product of continuous functions.

The actual construction of such algebras enjoying these optimal properties is due to Colombeau [9]. His theory of algebras of generalized functions gave a rigorous mathematical sense to the "heuristic" computations that were used in quantum field theory. In his theory Colombeau deals with generalized functions which are defined on any open subset $\Omega$ of $\mathbf{R}^{n}$ and are more general than the distributions on $\Omega$. From the viewpoint of differentiation these new generalized functions have the same properties as distributions. It is from the viewpoint of multiplication and nonlinear operations that these new generalized functions have properties completely different from those of distributions: any finite product of generalized functions is still generalized function and, more generally, the algebras of these generalized functions are closed with respect to many nonlinear operations, so that any finite product of distributions is a generalized function and not a distribution in general. However, these new generalized functions are very much related to the distributions in the sense that their definition may be considered as a natural evolution of Schwartz's definition of distribution.

In a few words, the differential Colombeau algebra $\mathscr{G}$ as a powerful tool for solving linear and nonlinear problems includes singularities (see [10]) and has almost the optimal properties while the problem of multiplication of Schwartz's distributions is concerned: it is an associative differential algebra of generalized functions and contains the algebra of smooth functions as a subalgebra; the distribution space $\mathscr{D}^{\prime}$ is linearly embedded in it as a subspace and the multiplication is compatible with the operations of differentiation and products with $C^{\infty}$-differentiable functions. The notion "association" in $\mathscr{G}$ is a faithful generalization of the equality 
of distributions and enables obtaining results in terms of distributions again.

Following this approach, in this paper we evaluate some products of distributions, as embedded in Colombeau algebra, in terms of associated distributions. Similar results can be found in [11]. The results obtained in this way can be reformulated as regularized products in the classical distribution theory.

\section{Colombeau Algebra}

In this section we will introduce basic notations and definitions from Colombeau theory.

Let $\mathbf{N}_{\mathbf{0}}$ be the set of nonnegative integers, that is, $\mathbf{N}_{\mathbf{0}}=$ $\mathbf{N} \cup\{0\}$. For $q \in \mathbf{N}_{\mathbf{0}}$ we denote

$$
\begin{array}{r}
A_{q}(\mathbf{R})=\left\{\varphi(x) \in \mathscr{D}(\mathbf{R}) \mid \int_{\mathbf{R}} \varphi(x) d x=1,\right. \\
\left.\int_{\mathbf{R}} x^{j} \varphi(x) d x=0, j=1, \ldots, q\right\},
\end{array}
$$

where $\mathscr{D}(\mathbf{R})$ is the space of all $C^{\infty}$ functions $\varphi: \mathbf{R} \rightarrow \mathbf{C}$ with compact support. The elements of the set $A_{q}(\mathbf{R})$ are called test functions. It is obvious that $A_{1} \supset A_{2} \supset A_{3} \ldots$... Also, $A_{k} \neq \emptyset$ for all $k \in \mathbf{N}$. For $\varphi \in A_{q}(\mathbf{R})$ and $\varepsilon>0$ it is denoted, $\varphi_{\varepsilon}=$ $((1 / \varepsilon) \varphi)(x / \varepsilon)$ and $\check{\varphi}(x)=\varphi(-x)$.

Wanting to obtain an algebra containing the space of distributions, whose elements could be multiplied and differentiated as well as $C^{\infty}$ functions, Colombeau started with $\mathscr{E}(\mathbf{R})$, the algebra of functions $f(\varphi, x): A_{0}(\mathbf{R}) \times \mathbf{R} \rightarrow \mathbf{C}$ that are infinitely differentiable with respect to the second variable, $x$. The embedding of distributions into such an algebra must be done in a way that the embedding of $C^{\infty}$ functions will be identity. Let $f$ and $g$ be $C^{\infty}$ functions. Taking the sequence $\left(f * \varphi_{\varepsilon}\right)_{\varepsilon>0}$, which converges to $f$ in $\mathscr{D}^{\prime}$, as a representative of $f$, we obtain an embedding of $\mathscr{D}^{\prime}$ into $\mathscr{E}(\mathbf{R})$. So, if we consider $f$ and $g$ as distributions, we look at the sequences $\left(f * \varphi_{\varepsilon}\right)_{\varepsilon>0}$ and $\left(g * \varphi_{\varepsilon}\right)_{\varepsilon>0}$. The product of $f$ and $g$ as distributions does not always coincide with their classical product considered as a distribution; that is,

$$
\left(f * \varphi_{\varepsilon}\right)\left(g * \varphi_{\varepsilon}\right) \neq(f g) * \varphi_{\varepsilon} .
$$

The idea therefore is to find an ideal $\mathscr{I}[\mathbf{R}]$ such that this difference will vanish in the resulting quotient. In order to determine $\mathscr{I}[\mathbf{R}]$ it is obviously enough to find an ideal containing the differences $\left(\left(f * \varphi_{\varepsilon}\right)-f\right)_{\varepsilon>0}$.

Expanding the last term in a Taylor's series and having in mind properties of $\varphi(x)$ as an element of $A_{q}(\mathbf{R})$ we can see that it will vanish faster than any power of $\mathcal{\varepsilon}$, uniformly on compact sets, in all derivatives. The set of these differences will not be an ideal in $\mathscr{E}(\mathbf{R})$ but in a set of a sequences whose derivatives are bounded uniformly on compact sets by negative power of $\varepsilon$. These sequences are called "moderate" sequences and the set containing them is denoted by $\mathscr{E}_{M}[\mathbf{R}]$.

Finally, the generalized functions of Colombeau are elements of the quotient algebra

$$
\mathscr{G} \equiv \mathscr{G}(\mathbf{R})=\frac{\mathscr{E}_{M}[\mathbf{R}]}{\mathscr{I}[\mathbf{R}]},
$$

where, as explained before, $\mathscr{E}_{M}[\mathbf{R}]$ is the subalgebra of "moderate" functions such that for each compact subset $K$ of $\mathbf{R}$ and any $p \in \mathbf{N}_{\mathbf{0}}$ there is a $q \in \mathbf{N}$, such that, for each $\varphi \in A_{q}(\mathbf{R})$, there are $c>0, \eta>0$ and it holds

$$
\sup _{x \in K}\left|\partial^{p} f\left(\varphi_{\varepsilon}, x\right)\right| \leq c \varepsilon^{-q},
$$

for $0<\varepsilon<\eta$ and $\mathscr{I}[\mathbf{R}]$ is an ideal of $\mathscr{C}_{M}[\mathbf{R}]$ consisting of all functions $f(\varphi, x)$ such that for each compact subset $K$ of $\mathbf{R}$ and any $p \in \mathbf{N}_{\mathbf{0}}$ there is a $q \in \mathbf{N}$ such that for every $r \geq q$ and each $\varphi \in A_{r}(\mathbf{R})$ there are $c>0, \eta>0$ and it holds:

$$
\sup _{x \in K}\left|\partial^{p} f\left(\varphi_{\varepsilon}, x\right)\right| \leq c \varepsilon^{r-q}
$$

for $0<\varepsilon<\eta$. Elements of $\mathscr{I}[\mathbf{R}]$ are also known as "null" functions or "negligible" functions.

The Colombeau algebra $\mathscr{G}(\mathbf{R})$ contains the distributions on $\mathbf{R}$ canonically embedded as a $\mathbf{C}$-vector subspace by the map:

$$
\begin{aligned}
i: \mathscr{D}^{\prime}(\mathbf{R}) \longrightarrow \mathscr{G}(\mathbf{R}): u \longrightarrow \widetilde{u} \\
\quad=\left\{\tilde{u}(\varphi, x)=(u * \check{\varphi})(x): \varphi \in A_{q}(\mathbf{R})\right\},
\end{aligned}
$$

where $*$ denotes the convolution product of two distributions and is given by

$$
(f * g)(x)=\int_{\mathbf{R}} f(y) g(x-y) d y .
$$

According to the above, we can also write: $\widetilde{u}(\varphi, x)=$ $\langle u(y), \varphi(y-x)\rangle$, where $\langle u, \varphi\rangle$ denotes the integral $\int_{\mathbf{R}} u(x) \varphi(x) d x$.

An element $f \in \mathscr{G}$ (a generalized function of Colombeau) is actually an equivalence class $[f]=\left[f_{\varepsilon}+\mathscr{I}\right]$ of an element $f_{\varepsilon} \in \mathscr{E}_{M}$ which is called representative of $f$. Multiplication and differentiation of generalized functions are performed on arbitrary representatives of the respective generalized functions.

With the next two definitions, we introduce the notion of "association".

Definition 1. Generalized functions $f, g \in \mathscr{G}(\mathbf{R})$ are said to be associated and denoted by $f \approx g$, if for some representatives $f\left(\varphi_{\varepsilon}, x\right)$ and $g\left(\varphi_{\varepsilon}, x\right)$ and arbitrary $\psi(x) \in \mathscr{D}(\mathbf{R})$ there is a $q \in \mathbf{N}_{\mathbf{0}}$ such that for any $\varphi(x) \in A_{q}(\mathbf{R})$

$$
\lim _{\varepsilon \rightarrow 0_{+}} \int_{\mathbf{R}}\left|f\left(\varphi_{\varepsilon}, x\right)-g\left(\varphi_{\varepsilon}, x\right)\right| \psi(x) d x=0 .
$$

Definition 2. A generalized function $f \in \mathscr{G}$ is said to admit some $u \in \mathscr{D}^{\prime}(\mathbf{R})$ as "associated distribution", denoted by $f \approx$ $u$, if for some representative $f\left(\varphi_{\varepsilon}, x\right)$ of $f$ and any $\psi(x) \in$ $\mathscr{D}(\mathbf{R})$ there is a $q \in \mathbf{N}_{\mathbf{0}}$ such that for any $\varphi(x) \in A_{q}(\mathbf{R})$

$$
\lim _{x \rightarrow 0_{+}} \int_{\mathbf{R}} f\left(\varphi_{\varepsilon}, x\right) \psi(x) d x=\langle u, \psi\rangle .
$$

These definitions are independent of the representatives chosen and the distribution associated, if it exists, is unique. The association is a faithful generalization of the equality of distributions. 
Multiplying two distributions in $\mathscr{G}$ as a result it is in general obtained a generalized function which may not always be associated with the third distribution. By Colombeau product of distributions it is meant the product of their embedding in $\mathscr{G}$ whenever the result admits an associated distribution. If the regularized model product of two distributions exists, then their Colombeau product also exists and it is the same with the first one. The relation $f \approx u$ is asymmetric; the distribution $u$ stands on the r.h.s.; the relation $f \approx \widetilde{u}$ is an equivalent relation in $\mathscr{G}$ so it is symmetric in $\mathscr{G}$ and it can also be written as $f-\tilde{u} \approx 0$. As a final conclusion of this introduction we have a fact that we operate with the elements of $\mathscr{G}$ exactly the same as with the $C^{\infty}$-functions, because we actually operate with their representatives which are $C^{\infty}$ functions.

\section{Results on Some Products of Distributions}

Recently, Li in [12] considered several results concerning commutative product of distributions and proved that

$$
x^{-1} \cdot \delta(x)=-\frac{1}{2} \delta^{\prime}(x) .
$$

In [13] it was proved that for any $p \in \mathbf{N}$ the product of the generalized functions $\widetilde{x^{-p}}$ and $\widetilde{\delta^{-1}(x)}$ in $\mathscr{G}(\mathbf{R})$ holds:

$$
\widetilde{x^{-p}} \cdot \widetilde{\delta^{(p-1)}(x)} \approx \frac{(-1)^{|p|}(p-1) !}{2(2 p-1) !} \delta^{(2 p-1)}(x) \text {. }
$$

Also in [14] the product of the generalized functions $\widetilde{\ln |x|}$ and $\widetilde{\delta^{(p-1)}(x)}$ in $\mathscr{G}(\mathbf{R})$ was considered and it was proved that

$$
\widetilde{\ln |x|} \cdot \widetilde{\delta^{(p-1)}(x)} \approx \frac{(-1)^{p}}{p} \delta^{(p-1)}(x) .
$$

In this paper we will make a generalization of (11), first for the one-dimensional case. Products of the form $x^{-k} \delta^{(p)}(x)$ are useful in quantum renormalization theory in Physics.

Theorem 3. The product of the generalized functions $\widetilde{x_{+}^{-k}}$ and $\widetilde{\delta^{(p)}(x)}$ for $k=1,2, \ldots$ and $p=0,1,2, \ldots$ in $\mathscr{G}(\mathbf{R})$ admits associated distributions and it holds

$$
\widetilde{x_{+}^{-k}} \cdot \widetilde{\delta^{(p)}(x)} \approx \frac{(-1)^{k} k \cdot p !}{(p+k+1) !} \delta^{(k+p)}(x)
$$

Proof. For given $\varphi \in A_{0}(\mathbf{R})$ we suppose that $\operatorname{supp} \varphi(x) \subseteq$ $[-l, l]$, without loss of generality. By definition we have $x_{+}^{-k}=$ $\left((-1)^{k-1} /(k-1) !\right)\left(d^{k}\right) /\left(d x^{k}\right)(\ln x)$. Then using the embedding rule and the substitution $t=(y-x) / \varepsilon$ we have the representatives of the distribution $x_{+}^{-k}$ in Colombeau algebra:

$$
\begin{aligned}
\widetilde{x_{+}^{-k}}\left(\varphi_{\varepsilon}, x\right) & =\frac{(-1)^{2 k-1}}{(k-1) ! \varepsilon^{k+1}} \int_{x}^{x+\varepsilon l} \ln y \varphi^{(k)}\left(\frac{y-x}{\varepsilon}\right) d y \\
& =\frac{(-1)^{2 k-1}}{(k-1) ! \varepsilon^{k}} \int_{0}^{l} \ln (x+\varepsilon t) \varphi^{(k)}(t) d t
\end{aligned}
$$

Differentiating in $\mathscr{D}^{\prime}(\mathbf{R})$, we get the representative of $\widetilde{\delta^{(p)}(x)}$ :

$$
\delta^{\left(\widetilde{p)}\left(\varphi_{\varepsilon}, x\right)\right.}=\frac{(-1)^{p}}{\varepsilon^{p+1}} \varphi^{(p)}\left(-\frac{x}{\varepsilon}\right) .
$$

Then, for any $\psi(x) \in \mathscr{D}(\mathbf{R})$ we have

$$
\begin{aligned}
& \left.\left.\left\langle x_{+}^{-\bar{k}\left(\varphi_{\varepsilon}\right.}, x\right) \cdot \delta^{(\bar{p})\left(\varphi_{\varepsilon}\right.}, x\right), \psi(x)\right\rangle \\
& \left.\left.=\int_{0}^{\infty} x^{-\widetilde{k}\left(\varphi_{\varepsilon}\right.}, x\right) \delta^{(p)\left(\varphi_{\varepsilon}\right.}, x\right) \psi(x) d x \\
& =\frac{(-1)^{2 k+p-1}}{(k-1) ! \varepsilon^{p+k+1}} \\
& \times \int_{0}^{l \varepsilon}\left(\int_{0}^{l} \ln (x+\varepsilon t) \varphi^{(k)}(t) d t\right) \\
& \times \varphi^{(p)}\left(-\frac{x}{\varepsilon}\right) \psi(x) d x \\
& =\frac{(-1)^{p}}{(k-1) ! \varepsilon^{p+k}} \int_{0}^{l} \varphi^{(p)}(u) \psi(-\varepsilon u) \\
& \times \int_{0}^{l} \ln |\varepsilon t-\varepsilon u| \varphi^{(k)}(t) d t d u,
\end{aligned}
$$

using the substitution $u=-x / \varepsilon$. By the Taylor theorem we have

$$
\psi(-\varepsilon \mathcal{\varepsilon})=\sum_{i=0}^{p+k} \frac{\psi^{(i)}(0)}{i !}(-\varepsilon \mathcal{u})^{i}+\frac{\psi^{(p+k+1)}(\eta u)}{(p+k+1) !}(-\varepsilon \eta)^{p+k+1}
$$

for $\eta \in(0,1)$. Using relation (17) in (16) and changing the order of integration we have

$$
\begin{aligned}
& \left\langle x_{+}^{-\widetilde{k}\left(\varphi_{\varepsilon}, x\right)} \cdot \delta^{\left(\widetilde{p)}\left(\varphi_{\varepsilon}, x\right)\right.}, \psi(x)\right\rangle \\
& =\sum_{i=0}^{p+k} \frac{(-1)^{p+i} \psi^{(i)}(0)}{i !(k-1) ! \varepsilon^{p+k-i}} J_{i}+O(\varepsilon) \cdot \ln (\varepsilon),
\end{aligned}
$$

where $J_{i}=\int_{0}^{l} \varphi^{(k)}(t) d t \int_{0}^{l} \ln |\varepsilon t-\varepsilon u| u^{i} \varphi^{(p)}(u) d u$ and $i=$ $0,1, \ldots, p+k$.

Next using integration by part we have

$$
\begin{aligned}
J_{i}= & \int_{0}^{l} \varphi^{(k)}(t) d t \int_{0}^{l} \ln |\varepsilon t-\varepsilon u| u^{i} \varphi^{(p)}(u) d u \\
= & \frac{1}{i+1} \int_{0}^{l} \varphi^{(k)}(t) d t \int_{0}^{l} \ln |\varepsilon t-\varepsilon u| \varphi^{(p)} \\
& \times(u) d\left(u^{i+1}-t^{i+1}\right) \\
= & -\frac{1}{i+1} \int_{0}^{l} \varphi^{(k)}(t) d t \int_{0}^{l}\left(u^{i+1}-t^{i+1}\right) \\
& +\frac{1}{i+1} \int_{0}^{l} \varphi^{(k)}(t) d t \int_{0}^{l} \frac{u^{i+1}-t^{i+1}}{u-t} \varphi^{(p)}(u) d u .
\end{aligned}
$$


The first term above is zero, and we have

$$
\begin{aligned}
(i+1) J_{i}= & \sum_{s=0}^{i} \int_{0}^{l} t^{s} \varphi^{(k)}(t) d t \\
& \times \int_{0}^{l} u^{i-s} \varphi^{(p)}(u) d u=\sum_{s=0}^{i} J_{s, k} \cdot J_{i-s, p},
\end{aligned}
$$

where $J_{a, b}=\int_{0}^{l} v^{a} \varphi^{(b)}(v) d v$. The integral $J_{a, b}$ is nonzero only if $a=b$, and its value is $J_{a, a}=(-1)^{a} a$ !. Thus the only nonzero term in the sum above is obtained when $i=k+p$; that is, $J_{k+p}=\left(J_{k, k} \cdot J_{p, p}\right) /(p+k+1)=\left((-1)^{p+k} p ! k !\right) /(p+k+1)$. Consider

$$
\begin{aligned}
& \left.\left\langle x_{+}^{-\widetilde{k}\left(\varphi_{\varepsilon}, x\right)} \cdot \delta^{(\widetilde{p})\left(\varphi_{\varepsilon}\right.}, x\right), \psi(x)\right\rangle \\
& =\frac{(-1)^{p} k \cdot p !}{(p+k+1) !} \psi^{(k+p)}(0)+O(\varepsilon) \\
& =\frac{(-1)^{k} k \cdot p !}{(p+k+1) !}\left\langle\delta^{(k+p)}(x), \psi(x)\right\rangle+O(\varepsilon) \text {. }
\end{aligned}
$$

Therefor, passing to the limit, as $\varepsilon \rightarrow 0$, we obtain (13) proving the theorem.

Next, to extend our main result in $\mathscr{G}\left(\mathbf{R}^{\mathbf{m}}\right)$ we need the following lemma proved in [15].

Lemma 4. Let $u$ and $v$ be distributions in $\mathscr{D}\left(\mathbf{R}^{\mathbf{m}}\right)$ such that $u(x)=\prod_{i=1}^{m} u^{i}\left(x_{i}\right)$ and $v(x)=\prod_{i=1}^{m} v^{i}\left(x_{i}\right)$ with each $u^{i}$ and $v^{i}$ in $\mathscr{D}(\mathbf{R})$ and suppose that their embedding in $\mathscr{G}(\mathbf{R})$ satisfy $\widetilde{u^{i}} \cdot \widetilde{v^{i}} \approx \omega^{i}$, for $i=1,2, \ldots, m$. Then $\tilde{u} \cdot \widetilde{v} \approx \omega$, where $\omega(x)=$ $\prod_{i=1}^{m} \omega^{i}\left(x_{i}\right)$ is in $\mathscr{D}\left(\mathbf{R}^{\mathbf{m}}\right)$.

Theorem 5. The product of the generalized functions $\widetilde{x_{+}^{-k}}$ and $\widetilde{\delta^{(p)}(x)}$ for $k=1,2, \ldots$ and $p=0,1,2, \ldots$ in $\mathscr{G}\left(\mathbf{R}^{\mathbf{m}}\right)$ admits associated distributions and it holds

$$
\widetilde{x_{+}^{-k}} \cdot \widetilde{\delta^{(p)}(x)} \approx \frac{(-1)^{k} k \cdot p !}{(p+k+1) !} \delta^{(k+p)}(x) .
$$

Proof. In this case, due to the tensor product structure of the distributions that are considered, we can apply previous lemma and we have

$$
\begin{aligned}
& \widetilde{x_{+}^{-k}} \cdot \widetilde{\delta^{(p)}(x)} \\
&=\prod_{i=1}^{m} \widetilde{x_{i+}^{-k_{i}}} \cdot \widetilde{\delta^{\left(p_{i}\right)}\left(x_{i}\right)} \\
& \approx \prod_{i=1}^{m} \frac{(-1)^{p_{i}} k_{i} \cdot p_{i} !}{\left(p_{i}+k_{i}+1\right) !} \delta^{\left(k_{i}+p_{i}\right)}\left(x_{i}\right) \\
&=\frac{(-1)^{k} k \cdot p !}{(p+k+1) !} \delta^{(k+p)}(x),
\end{aligned}
$$

which completes the proof of the theorem.

\section{Conflict of Interests}

The authors declare that there is no conflict of interests regarding the publication of this paper.

\section{Acknowledgments}

This work is supported by the research project "Functional Spaces, Topological and Statistical Aspects and Their Application in Electrical Engineering" and financed by State University “Goce Delce”, Stip, Macedonia.

\section{References}

[1] S. Gasiorawics, Elementary Particle Physics, John Wiley and sons, New York, NY, USA, 1966.

[2] M. Oberguggenberger, Multiplication of Distributions and Applications to Partial Differential Equations, Longman, Essex, UK, 1992.

[3] H. König, "Neue Begründung der Theorie der "Distributionen" von L. Schwartz," Mathematische Nachrichten, vol. 9, no. 3, pp. 129-148, 1953.

[4] P. Antosik, J. Mikusinski, and R. Sigorski, Theory of Distributions. The Sequential Approach, Elsevier, Amsterdam, The Netherlands, 1973.

[5] B. Fisher, "The product of distributions," The Quarterly Journal of Mathematics, vol. 22, pp. 291-298, 1971.

[6] B. Fisher, "On defining the product of the distributions," Mathematische Nachrichten, vol. 108, pp. 117-127, 1982.

[7] C. L. Zhi and B. Fisher, "Several products of distributions on $\mathbf{R}^{m}$," Proceedings of the Royal Society A, vol. 426, no. 1871, pp. 425-439, 1989.

[8] L. Schwartz, "Sur l'impossibilité de la multiplication des distributions," Comptes Rendus de l'Académie des Sciences, vol. 239, pp. 847-848, 1954.

[9] J. F. Colombeau, New Generalized Functions and Multiplication of Distributions, North-Holland Mathematics Studies, NorthHolland Publishing, Amsterdam, The Netherlands, 1984.

[10] B. Jolevska-Tuneska, A. Takači, and E. Özçag, "On differential equations with nonstandard coefficients," Applicable Analysis and Discrete Mathematics, vol. 1, no. 1, pp. 1-8, 2007.

[11] B. Jolevska-Tuneska and T. Atanasova-Pacemska, "Further results on Colombeau product of distributions," International Journal of Mathematics and Mathematical Sciences, vol. 2013, Article ID 918905, 5 pages, 2013.

[12] C. K. Li, "Several results on the commutative neutrix product of distributions," Integral Transforms and Special Functions, vol. 18, no. 8, pp. 559-568, 2007.

[13] B. P. Damyanov, "Multiplication of Schwartz distributions and Colombeau generalized functions," Journal of Applied Analysis, vol. 5, no. 2, pp. 249-260, 1999.

[14] M. Miteva and B. Jolevska-Tuneska, "Some results on Colombeau product of distributions," Advances in Mathematics, vol. 1, no. 2, pp. 121-126, 2012.

[15] B. Damyanov, "Results on Colombeau product of distributions," Commentationes Mathematicae Universitatis Carolinae, vol. 38, no. 4, pp. 627-634, 1997. 


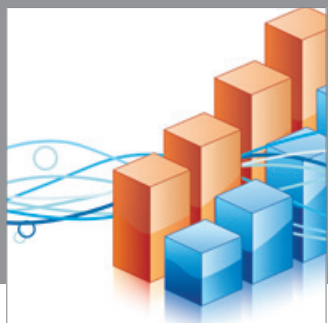

Advances in

Operations Research

mansans

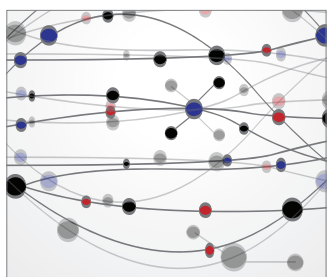

The Scientific World Journal
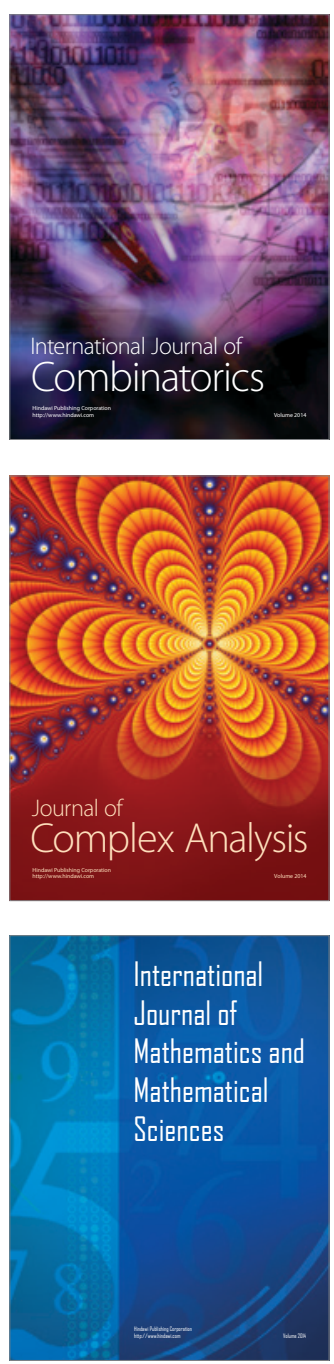
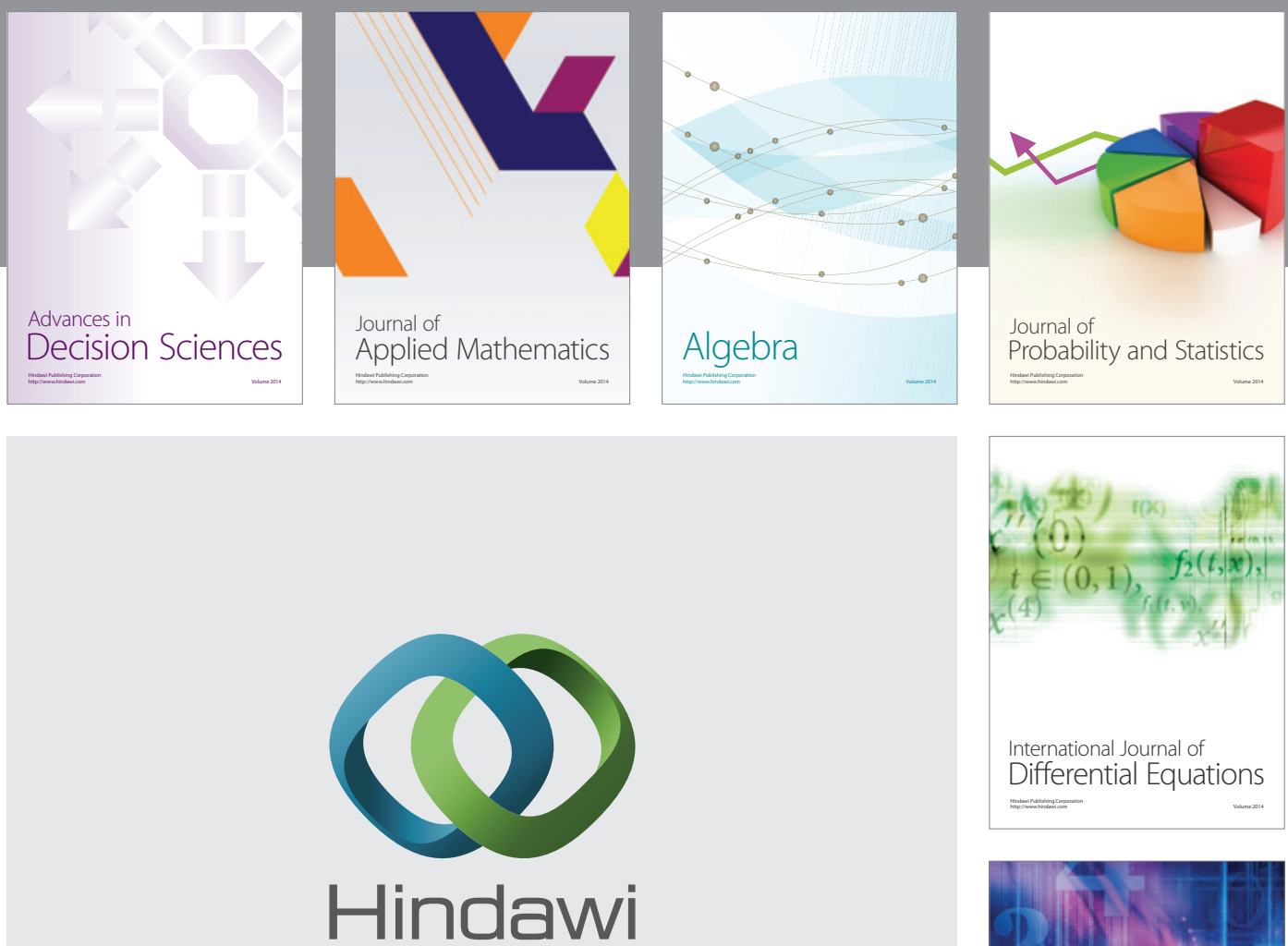

Submit your manuscripts at http://www.hindawi.com
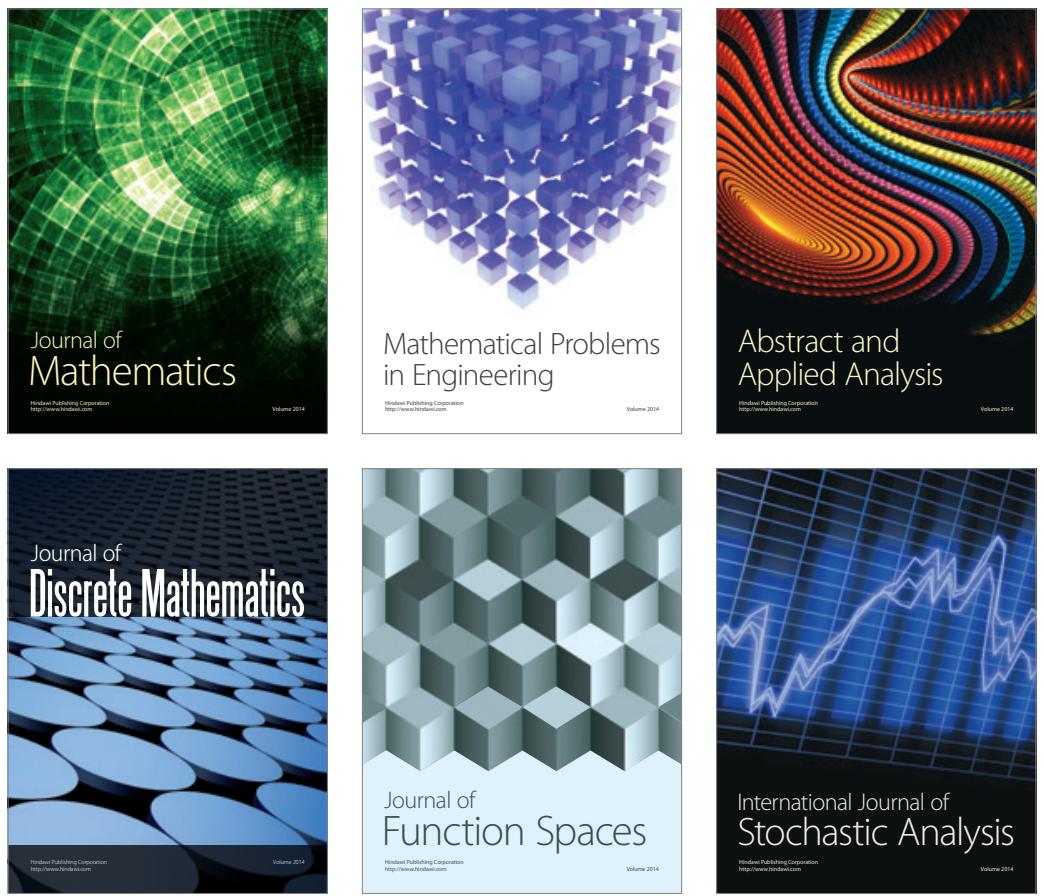

Journal of

Function Spaces

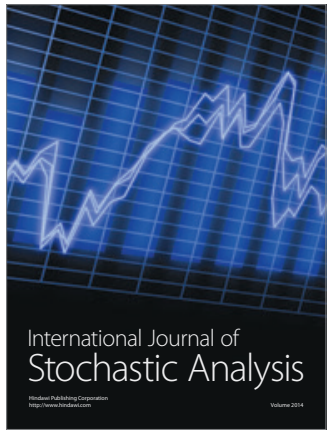

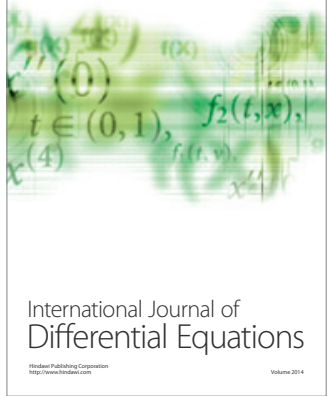
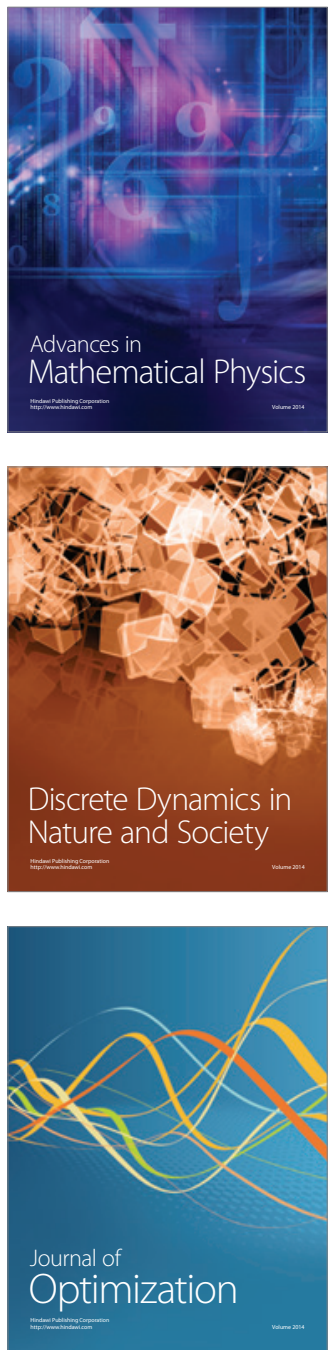